\title{
Cutaneous anthrax leading to corneal scarring from cicatricial ectropion
}

\author{
DAVID YORSTON' AND ALLEN FOSTER ${ }^{2}$ \\ From the 'Mvumi Hospital, PO Dodoma, Tanzania, and the ${ }^{2}$ Institute of Ophthalmology, London
}

SUMMARY Eleven patients with cutaneous anthrax of the eyelids are presented. The complications were cicatricial ectropion (eight patients), resulting in corneal scarring (three patients). The ectropion was corrected by full thickness postauricular skin grafts, with good results. The predilection of this infection for the eyelids of young children and a seasonal variation suggest that a vector may be involved in transmission.

Anthrax is an infectious disease of the skin and respiratory or gastrointestinal tracts, caused by the Gram-positive sporing Bacillus anthracis. The spores of $B$. anthracis are notoriously hardy and long-lived. They can resist temperatures of over $100^{\circ} \mathrm{C}$ for prolonged periods, and are resistant to drying. They can remain dormant but potentially infective for over 20 years. Tissue damage in the host is caused by a complex exotoxin which damages the capillary endothelium and causes inhibition of phagocytosis.'

Anthrax is a zoonosis, the main reservoir of infection being domestic herbivores such as cattle, goats, and sheep. Man can become infected through direct contact with contaminated skins; inhalation of spores from contaminated animal products, such as wool fibres or bone meal; or from eating infected meat. In addition it has been suggested that insects may act as vectors of infection from an infected animal to man.

The first sign of cutaneous anthrax is severe and massive oedema round the site of infection. The oedema can spread from a single lesion to involve the whole trunk. Oedema of the neck may be of such severity as to obstruct the airway, necessitating tracheostomy. The oedema is characteristically nonpitting, and has been described as 'gelatinous'. At this early stage translucent epidermal bullae and vesicles often surround the lesion - the so-called 'pearly wreath'. As the oedema subsides, a characteristic pustule and area of tissue necrosis develops at the site of inoculation (Fig. 1). This forms a black slough, known as the eschar or charbon (Fig. 2).
After two to four weeks the eschar sloughs away, leaving an area of exposed granulation tissue, which heals with scarring. If the lesion is on the eyelids, scarring and contracture occur and may lead to cicatricial ectropion. ${ }^{2}$

Before the advent of antibiotics, and today in rural areas of the developing world where antibiotics are often not available, cutaneous anthrax may lead to septicaemia, which has a mortality rate of $20-30 \%$. Parenteral penicillin in high doses is still the recommended first line treatment.'

This paper reports a series of 11 patients with periocular anthrax and its complications seen at Mvumi Hospital; Tanzania. Mvumi Hospital is an Anglican mission hospital 26 miles south-east of Dodoma in the centre of Tanzania. This is one of the driest, least fertile, and poorest regions of the country. The local people, the Wagogo, are mixed farmers, growing millet and keeping large herds of cattle and goats. They have a high incidence of eye disease, particularly trachoma, and the prevalence of blindness (binocular visual acuity less than $3 / 60$ ) is $1.6 \% .^{3}$

\section{Patients}

Between January 1984 and June 198811 patients with periocular anthrax or its sequelae were admitted to the Mvumi Hospital Eye Unit (Table 1). Six presented with acute anthrax of the eyelids and five with late sequelae. In patients who presented late the diagnosis was based on the presence of characteristic scarring and a history of severe swelling and black eschar formation prior to the development of the ectropion. 


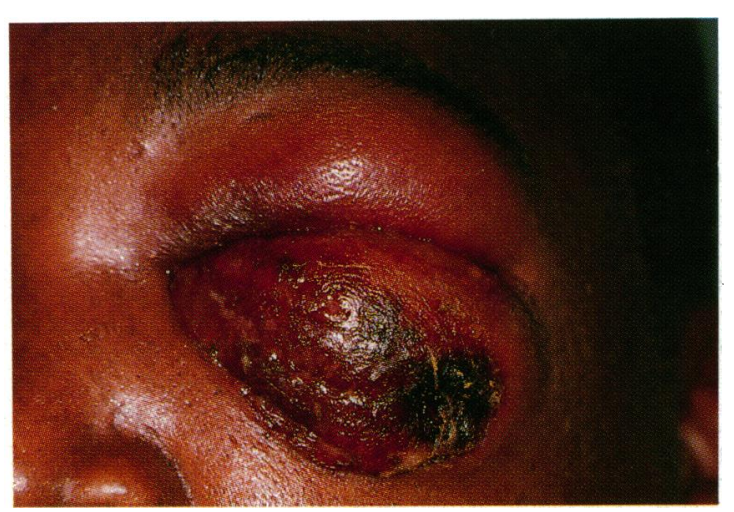

Fig. 1 Severe eyelid oedema and early eschar formation in cutaneous anthrax.

The main complications seen were cicatricial ectropion (eight patients) and corneal scar secondary to exposure from ectropion (three patients).

Six females and five males were affected. Seven patients were children. The age range was 4 months to 45 years at the time of infection. The upper lid was affected in six patients and the lower lid in five. There appeared to be a seasonal trend, with the onset of infection being more common during the rainy season.

The six patients with acute anthrax were treated with high doses of parenteral penicillin. None developed anthrax septicaemia. Three developed ectropion, and three healed with scarring but without ectropion: these three had anthrax of the lower lid.

Eight patients with cicatricial ectropion (six upper and two lower) had their contractures released and a full thickness postauricular skin graft performed. In seven patients this was uncomplicated and a satisfactory resolution of the ectropion was achieved. In

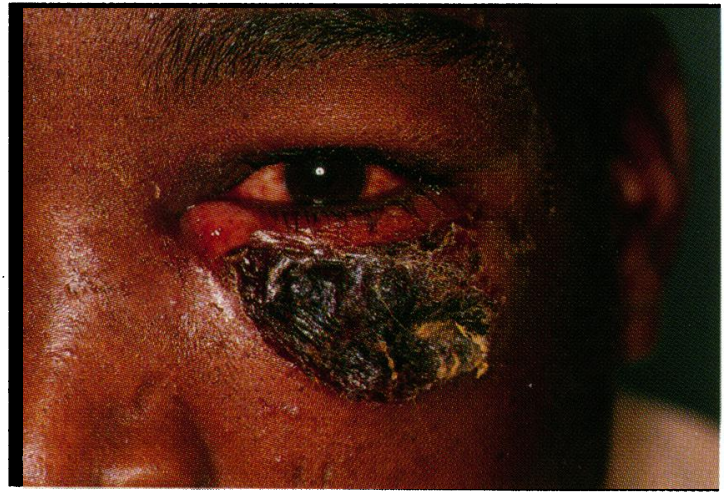

Fig. 2 The oedema has now resolved, leaving the black eschar.

one patient the skin graft became necrotic because of infection with a polyresistant staphylococcus, though correction of the ectropion was still satisfactory.

Corneal scar secondary to exposure occurred in three patients. These were all children who presented two to four years after the acute infection and had severe cicatricial ectropion, shortening of the upper eyelid, and exposure of the cornea.

\section{Discussion}

The main complication of periocular anthrax is cicatricial ectropion. This occurred in eight of 11 patients. Two out of five patients with lower lid anthrax developed ectropion, compared with six out of six for upper lid anthrax, suggesting that anthrax is more likely to cause ectropion when it affects the upper lid.

Exposure and corneal scar were associated with ectropion of the upper lid in three patients. The

Table 1 Clinical features of 11 patients with cutaneous anthrax of the eyelid

\begin{tabular}{|c|c|c|c|c|c|c|c|c|}
\hline \multirow[b]{2}{*}{ Patient } & \multirow[b]{2}{*}{ Age } & \multirow[b]{2}{*}{ Sex } & \multirow[b]{2}{*}{ Lid } & \multicolumn{3}{|c|}{ Presentation } & \multicolumn{2}{|c|}{ Complications } \\
\hline & & & & Mode & $\begin{array}{l}\text { Interval from first } \\
\text { symptoms }\end{array}$ & $\begin{array}{l}\text { Month of first } \\
\text { symptoms }\end{array}$ & Ectropion & $\begin{array}{l}\text { Corneal } \\
\text { Scar }\end{array}$ \\
\hline 1 & 25 & $\mathbf{M}$ & RL & Acute & 10 days & May & No & No \\
\hline 2 & 4 & $\mathbf{F}$ & LL & Acute & 1 week & June & No & No \\
\hline 3 & 45 & $\mathbf{F}$ & $\mathbf{L L}$ & Acute & 1 week & Jan & No & No \\
\hline 4 & 4 months & $\mathrm{F}$ & RL & Acute & 2 days & Nov & Yes & No \\
\hline 5 & 2 & $\mathbf{F}$ & RU & Acute & 1 week & Jan & Yes & No \\
\hline 6 & 26 & $F$ & RU & Acute & 2 weeks & Jan & Yes & No \\
\hline 7 & 3 & $\mathbf{M}$ & LU & Late & 3 months & April & Yes & No \\
\hline 8 & 26 & $\mathbf{M}$ & RL & Late & Unknown & Unknown & Yes & No \\
\hline 9 & $21 / 2$ & $\mathbf{F}$ & RU & Late & $2 \mathrm{yr}$ & Unknown & Yes & Yes \\
\hline 10 & 5 & $\mathbf{M}$ & RU & Late & Unknown & Unknown & Yes & Yes \\
\hline 11 & 12 & $\mathbf{M}$ & LU & Late & $4 \mathrm{yr}$ & Unknown & Yes & Yes \\
\hline
\end{tabular}

$R L=$ right lower. $L L=$ left lower. $R U=$ right upper. $L U=$ left upper. 
corneal scar was considered blinding in the patients with this complication, though two were too young to have their visual acuity measured. Since the six patients with upper lid ectropion all had a good result following skin grafting (that is a complete resolution of the ectropion and good lid closure), it seems probable that these corneal scars could have been prevented by earlier diagnosis, referral, and skin grafting. Three out of the seven children with periocular anthrax were blind in the affected eye.

Anthrax in this series appears to be more common during the rainy season than the dry, which is in accordance with a study from Zimbabwe, ${ }^{4}$ though a study in the Gambia found the highest incidence during the dry season." The seasonal variation parallels that of other vector borne diseases in the area, such as malaria and trachoma. It has been presumed that the most likely way of acquiring infection is direct contact with infected skins, though this would be unlikely to cause a seasonal variation.

During the period when six patients presented with acute periocular anthrax only two patients were admitted with anthrax of other parts of the body. This finding is difficult to explain if infection is acquired from direct contact with animal skins, which are in contact with the whole body when used as bedding. Moreover, if the main route of infection was contact with infected animals or animal products, it is surprising that over half of our patients were under 6 years old, as this group of the population has very little contact with livestock, which are cared for by older children and adults.

Although direct transmission from infected animal products, particularly skins, probably does occur, the predilection for the eyelids of children suggests other possible modes of transmission. Flies are commonly found on the face and round the eyes of small children, who consequently frequently rub their eyes. It is possible that the anthrax bacillus is transferred to the children's eyelids either by flies or by self-contamination from their own fingers after handling contaminated animal skins used for bedding. The survey of anthrax in Zimbabwe between January 1979 and March 1980 reported 6000 cases. The peak incidence was during the rainy season, and it was suggested that a vector may be responsible for transmission. They also found that anthrax was more likely to occur on exposed parts of the body. A number of their patients gave a history of being bitten or stung at the site of the lesion prior to the development of anthrax. ${ }^{+}$However, none of the patients in our study volunteered such a history.

\section{Conclusion}

Cutaneous anthrax in this small series was responsible for cicatricial ectropion, resulting in corneal scar and blindness in the affected eye. The ectropion can be corrected with good results by full-thickness skin grafting. There is anecdotal evidence that the disease has a predilection for the eyelids and occurs more commonly in children and during the rainy season. This suggests either that transmission occurs from rubbing the eyelids with contaminated fingers or possibly that an insect vector may spread the bacillus from animals to the children's eyelids.

We acknowledge the support of Christoffel Blindenmission of West Germany, which made this work possible.

\section{References}

1 Parry EHO. Principles of Medicine in Africa. 2nd ed. Oxford: Oxford Medical Publications, 1984: 308-13.

2 Duke-Elder SJ. System of Ophthalmology. Bristol: Kimpton, 1974: 13: (1): 97.

3 Prentice D, Turner G, Mmbaga BBO. Kongwa primary eye care report. New York: Helen Keller International, 1986.

4 McKendrick DRA. Anthrax and its transmission to humans. Cent Afr J Med 1980); 26: 126-9.

5 Heyworth B, Ropp ME, Voos UG, Meinel HI, Darlow HM. Anthrax in the Gambia: an epidemiological study. Br Med J 1975; iv: $79-82$.

Accepted for publication 27 April 1989. 